\title{
Modelling the quark propagator
}

\author{
Patrick O. Bowman ${ }^{\mathrm{a} *}$, Urs M. Heller ${ }^{\mathrm{a}}$, Derek B. Leinweber ${ }^{\mathrm{b}}$ and Anthony G. Williams ${ }^{\mathrm{b}}$ \\ ${ }^{a}$ Department of Physics and CSIT, Florida State University, Tallahassee FL 32306-4120, USA \\ ${ }^{\mathrm{b}} \mathrm{CSSM}$ and Department of Physics and Mathematical Physics, University of Adelaide, Australia 5005
}

The quark propagator is at the core of lattice hadron spectrum calculations as well as studies in other nonperturbative schemes. We investigate the quark propagator with an improved staggered action (Asqtad) and an improved gluon action, which provides good quality data down to small quark masses. This is used to construct ansätze suitable for model hadron calculations as well as adding to our intuitive understanding of QCD.

\section{The lattice quark propagator}

The quark propagator is a fundamental quantity of QCD. Though gauge dependent, it manifestly displays dynamical chiral symmetry breaking, contains the chiral condensate and $\Lambda_{\mathrm{QCD}}$, and has been used to compute the running quark mass 11. Some model hadron calculations rely on ansätze for the quark propagator [2], yet on the lattice we have the opportunity to study it in a direct, nonperturbative fashion.

We use the "Asqtad" quark action [3], a highly improved staggered action that formally has no $\mathcal{O}\left(a^{2}\right)$ errors. We extend some earlier work [4] by also using an improved gluon action. We have calculated the quark propagator on three sets of configurations: $12^{3} \times 24$ and $16^{3} \times 32$ at $\beta=$ $4.60(a=0.125 \mathrm{fm})$ and $16^{3} \times 32$ at $\beta=4.38$ $(a=0.167 \mathrm{fm})$, each ensemble consisting of 100 configurations. The configurations were fixed to Landau gauge. Most results shown here are from the larger, finer lattice, where we used 8 quark masses: $m a=0.012,0.018,0.024,0.036,0.048$, $0.072,0.108,0.144$ (19 to $114 \mathrm{MeV}$ ).

In the (Euclidean) continuum, Lorentz invariance allows us to decompose the full quark propagator into Dirac vector and scalar pieces

$$
S^{-1}\left(p^{2}\right)=Z^{-1}\left(p^{2}\right)\left[i \gamma \cdot p+M\left(p^{2}\right)\right] .
$$

Asymptotic freedom means that, as $p^{2} \rightarrow \infty$, $S^{-1}\left(p^{2}\right) \rightarrow i \gamma \cdot p+m$, (the free propagator) where $m$ is the bare quark mass.

\footnotetext{
*Talk presented by POB.
}

From consideration of the tree-level form of our lattice action, we define the momentum variable

$$
q_{\mu} \equiv \sin \left(p_{\mu}\right)\left[1+\frac{1}{6} \sin ^{2}\left(p_{\mu}\right)\right]
$$

for the Asqtad action, where $p_{\mu}$ is the usual lattice momentum,

$$
p_{\mu}=\frac{2 \pi n_{\mu}}{a L_{\mu}} \quad n_{\mu} \in\left[\frac{-L_{\mu}}{4}, \frac{L_{\mu}}{4}\right) .
$$

By considering the propagator as a function of $q_{\mu}$ instead of $p_{\mu}$, we ensure that the lattice quark propagator has the correct tree-level form for $Z$ and hopefully better approximates its continuum behaviour. It has already been seen that this produces a significant improvement in the rotational symmetry of $Z$. This prescription does not, however, tell us how to treat the mass function and in the past it has been considered as a function of either $p$ or $q$ in different studies.

Figure 1 shows the quark mass function for the coarser lattice as a function of both $p$ and of $q . M$ clearly has less anisotropy when plotted against $q$. This issue has been studied for the overlap quark propagator and there the mass function appears to converge to the continuum limit most rapidly as a function of $p$ [5]. The optimal momentum variable must therefore be determined for each action.

We test for finite volume effects by comparing the quark propagator on the two $\beta=4.60$ lattices in Fig. 2. No sign of finite volume artefacts is seen in the mass function, but the infrared supression of $Z$ is stronger on the smaller lattice. 

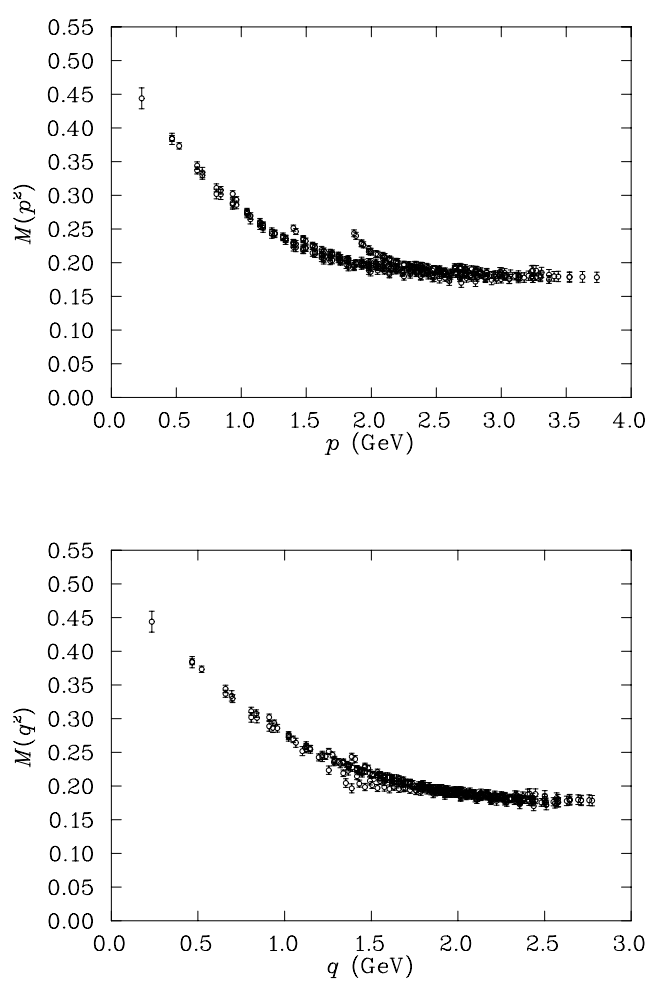

Figure 1. Quark mass function at $\beta=4.38(a \simeq$ $0.167 \mathrm{fm}$ ) for bare quark mass $m \simeq 114 \mathrm{MeV}$. Top figure uses the naive lattice momentum, while the bottom figure uses the momentum derived from the tree-level behaviour of the action.

\section{Parameterising the data}

The mass dependence of $M(q)$ can be seen in Fig. 3. Dynamical chiral symmetry breaking can be seen in the infrared enhancement of the mass function which, for small quark masses, is almost independent of the bare mass. For large quark mass, the function flattens off. In the case of the $Z$ function, dependence on the quark mass is very weak.

In the continuum, in the chiral limit, the asymptotic behaviour of the quark mass function is related to the chiral condensate [6]. We performed an extrapolation to the chiral limit using a quadratic fit in the quark mass to the cylinder cut data. A determination of the chiral condensate was then be made by fitting to the tail of

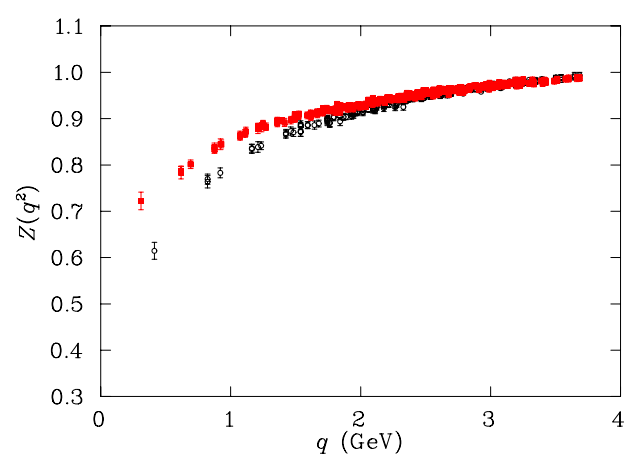

Figure 2. Quark $Z$ function for mass $m a=0.036$, at $\beta=4.60$. Comparison on $12^{3} \times 24$ lattice (open circles) and $16 \times 32$ lattice (solid squares). For $M(q)$ on the same lattices there are no discernible finite volume artefacts.

$M(q)$. We chose $2-3 \mathrm{GeV}(a p=1.27-1.9)$ for the fit region and set $\Lambda_{\mathrm{QCD}}$ at $691 \mathrm{MeV}$. We find $(-\langle\bar{\psi} \psi\rangle)^{1 / 3}=270(27) \mathrm{MeV}$; the quoted error is purely statistical.

A fit to each of the mass functions was done using the ansatz

$$
M\left(q^{2}\right)=\frac{c \lambda^{2(\alpha-1)}}{q^{2 \alpha}+\lambda^{2 \alpha}}+\widehat{m} .
$$

This is the same as the ansatz used in Ref. [4], but written slightly differently. We shall refer to it here as the "simple" ansatz. Fit results are shown in Table 1.

As before, we see that at small quark masses $\alpha \simeq 1.5$ is favoured, while it is near one for the heavier quarks. In this model, $\alpha$ is acting as a function of the bare mass, controlling the dynamical symmetry breaking. One fit is shown, for $M\left(q^{2}\right)$ at zero quark mass, in Fig. 3 .

We also investigated a more "complete" ansatz,

$$
\begin{gathered}
M\left(q^{2}\right)=c\left(\frac{A \lambda^{2(\alpha-1)}}{q^{2 \alpha}+\lambda^{2 \alpha}}+\frac{1}{q^{2}+\lambda^{2}}\left[\ln \frac{q^{2}+\lambda^{2}}{\Lambda_{\mathrm{QCD}}^{2}}\right]^{\gamma_{M}}\right) \\
+\frac{\widehat{m}}{\left[\ln \left(\left(q^{2}+\lambda^{2}\right) / \Lambda_{\mathrm{QCD}}^{2}\right)\right]^{d_{M}}},
\end{gathered}
$$

\footnotetext{
${ }^{1}$ This was computed by taking the ALPHA result of $\Lambda^{\overline{M S}}=239 \mathrm{MeV}$ 的 and converting it to the $\widetilde{M O M}$ scheme as described in Ref. [8].
} 
Table 1

Best-fit parameters for the simple ansatz, Eq. (4). Statistical uncertainties are determined by jackknife.

\begin{tabular}{ccccccc}
\hline$a m$ & $a^{3} c$ & $\lambda(\mathrm{MeV})$ & $\widehat{m}(\mathrm{MeV})$ & $\alpha$ & $M(0)(\mathrm{MeV})$ & $\chi^{2} /$ d.o.f. \\
\hline 0 & $0.040(3)$ & $719(50)$ & 0 & $1.47(11)$ & $302(33)$ & 0.72 \\
0.012 & $0.043(2)$ & $777(35)$ & $25(2)$ & $1.51(2)$ & $308(19)$ & 0.70 \\
0.018 & $0.044(2)$ & $795(28)$ & $37(2)$ & $1.50(2)$ & $311(13)$ & 0.65 \\
0.024 & $0.045(2)$ & $820(29)$ & $49(2)$ & $1.48(2)$ & $313(11)$ & 0.60 \\
0.036 & $0.046(2)$ & $802(27)$ & $70(3)$ & $1.31(1)$ & $353(10)$ & 0.53 \\
0.048 & $0.050(3)$ & $838(27)$ & $92(3)$ & $1.28(1)$ & $370(9)$ & 0.48 \\
0.072 & $0.059(2)$ & $946(29)$ & $138(5)$ & $1.30(8)$ & $397(12)$ & 0.44 \\
0.108 & $0.071(5)$ & $997(27)$ & $199(6)$ & $1.12(3)$ & $478(4)$ & 0.37 \\
0.144 & $0.090(9)$ & $1100(32)$ & $255(10)$ & $1.01(5)$ & $547(4)$ & 0.35 \\
\hline
\end{tabular}

which is an extension of the one used above that has the correct asymptotic behaviour. $A$ is dimensionless, $\widehat{m}$ is the RGI quark mass, $d_{M}$ is the anomalous dimension of the mass, $\gamma_{M}=d_{M}-1$, and $c$ can be related to the scalar condensate [6]. Unfortunately, the current data is insufficient to simultaneously determine $A$ and $c$. It is possible to determine $c$ separately from the asymptotic behaviour, as above, but the end product fits the data no better than the simple ansatz.

The work of UMH and POB was supported in part by DOE contract DE-FG02-97ER41022. DBL and AGW acknowledge financial support from the Australian Research Council.

\section{REFERENCES}

1. S. Aoki et al., Phys. Rev. Lett. 82, (1999) 4392; D. Becirevic et al., Phys. Rev. D 61, (2000) 114507.

2. M. Oettel and R. Alkofer, hep-ph/0204178.

3. K. Orginos, D. Toussaint and R.L. Sugar, Phys. Rev. D 60, (1999) 054503.

4. P.O. Bowman et al., Phys. Rev. D 66, (2002) 014505.

5. Talk by Jianbo Zhang, these proceedings.

6. F.D.R. Bonnet et al., Phys. Rev. D 65, (2002) 114503.

7. S. Capitani et al., Nucl. Phys. B 544, (1999) 669.

8. D. Becirevic et al., Phys. Rev. D 60, (1999) 094509.
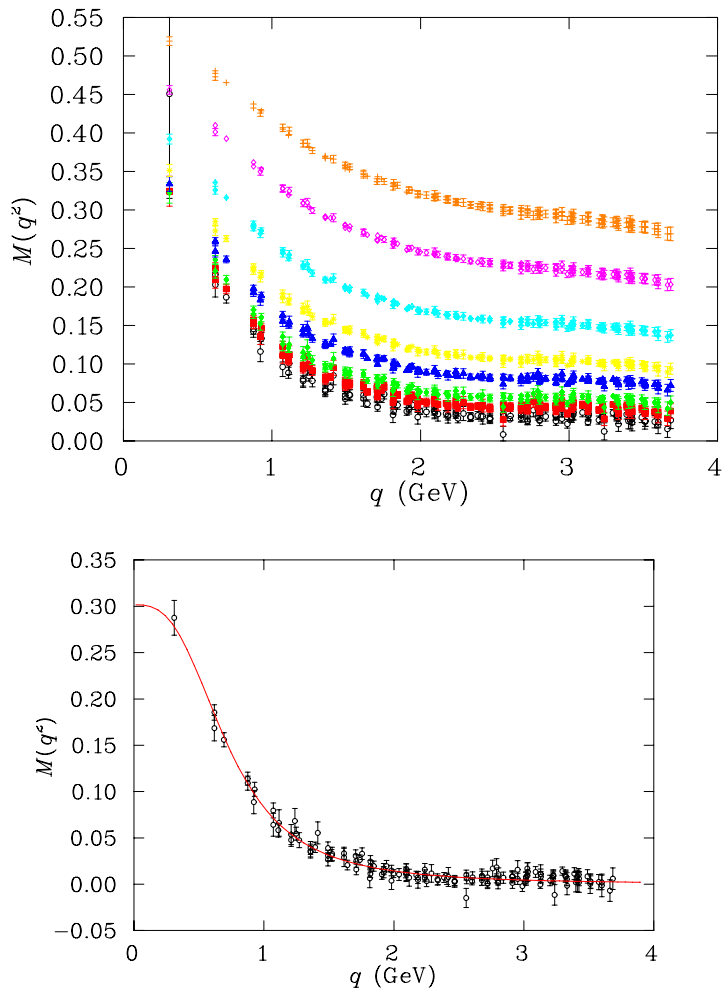

Figure 3. Quark mass function for all masses studied (top) and in the chiral limit (bottom) on the larger $\beta=4.60$ ensemble. In the chiral limit there is also a fit using the simple ansatz Eq. (位). 